\title{
MAMMOGRAFIE
}

\section{Zweifel am Nutzen von Screeningprogrammen}

Senkt das Mammografie-Screening die Zahl der Todesfälle durch Brustkrebs? In Dänemark, wo es solche Programme bereits seit 1991 gibt, ist dies einer neuen Studie zufolge nicht der Fall. Zwar ist die Brustkrebssterblichkeit in Regionen mit dem Röntgenscreening bei Frauen zwischen 55 und 74 Jahren um 1\% pro Jahr gesunken. Aber auch in Regionen ohne Rei- henuntersuchung ging die Mortalität in dieser Altersgruppe um $2 \%$ pro Jahr zurück. Bei Frauen, die noch zu jung für das Screening waren, nahm die Mammakarzinom-Sterblichkeit sogar um 5-6\% pro Jahr $a b$. Der Rückgang scheint also eher verminderte Risikofaktoren und verbesserte Therapiemöglichkeiten widerzuspiegeln. Brit Med J 2010;340:C1241

\section{HYGIENE}

\section{Was Keime wirklich ausknockt}

Mikrobiologen haben untersucht, welche Hygieneprodukte Keime von Händen am zuverlässigsten entfernen. Dazu wurden die Hände von 62 Probanden mit hartnäckigen (aber harmlosen) Bakterien und ebenfalls harmlosen Viren, die im Aufbau Erkältungsviren entsprachen, kontaminiert. Anschließend sollten die Probanden ihre Hände zehn Sekunden lang mit verschiedenen Reinigungsprodukten waschen. Da- nach wurde gemessen, wie viele Keime noch auf den Händen waren. Große Unterschiede fanden sich nicht. Testsieger war aber Seife plus Wasser. Die 10-SekundenKatzenwäsche beseitigte 90\% der Erreger. Eine alkoholhaltige Reinigungslotion - die eingesetzt werden kann, wenn gerade kein Wasser verfügbar ist - erbrachte mit 50\% das schlechteste Ergebnis. Mitteilung der American Society for Microbiology

\section{HERZINFARKT- UND SCHLAGANFALLRISIKO}

\section{BMI taugt nicht als Prädiktor}

Der BMI spielt keine Rolle für das Schlaganfall-, Herzinfarkt- oder Todesrisiko eines Menschen: Zu diesem Schluss kommt eine Studie aus München, in der 11000 Probanden drei bis acht Jahre beobachtet wurden. Zu Beginn der Studie wurden vier Parameter gemessen, deren prädiktive Kraft im Hinblick auf Herz-Kreislauf-Erkrankungen diskutiert wird: Bodymass-
Index (BMI), Taillenumfang (WC), das Verhältnis von Taillen- zu Hüftumfang (WHR) und der Quotient aus Taillenumfang und Körpergröße (WHtR). Am besten ließ sich das kardiovaskuläre Risiko mit dem WHtR abbilden. Der WHR war deutlich weniger, der BMI gar nicht aussagekräftig.

Pressemitteilung des Klinikums Innenstadt der LMU München; J Clin Endocrinol Metab, April 2010, 95(4)

\section{Zitat}

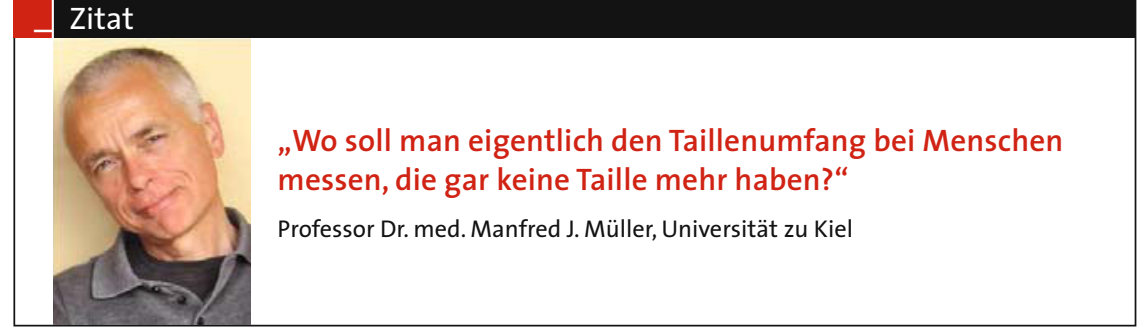

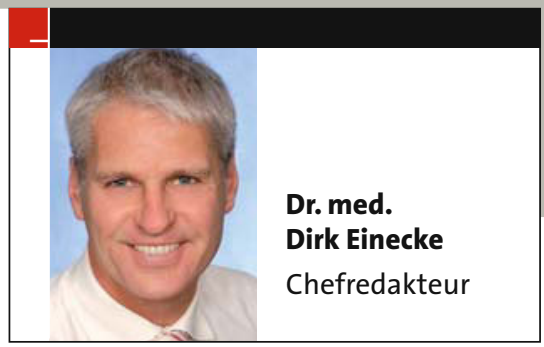

\section{NEUE METAANALYSE BESTÄTIGT}

\section{Primärprävention mit ASS nicht sinnvoll}

Bei Patienten, die (noch) keine vaskuläre Erkrankung haben, ist der Nutzen von ASS höchst fraglich. Zwar sinkt einer neuen Metaanalyse zufolge unter primärpräventiver ASS-Behandlung die Rate kardiovaskulärer Ereignisse relativ um 10\%. Wegen des geringen Ausgangsrisikos beträgt die absolute Risikoreduktion aber gerade mal $0,06 \%$. Das heißt, um ein vaskuläres Ereignis zu verhindern, müssen 1667 Patienten behandelt werden. Dem gegenüber steht die Zahl von 3333 Patienten, von denen einer infolge der ASS-Gabe eine extrakranielle Blutung erleiden wird. Selbst bei Diabetikern zeigte die Metaanalyse keinen Einfluss der ASS-Primärprävention auf das kardiovaskuläre 10-Jahres-Risiko.

Das J et al. ACC-Jahrestagung, März 2010

\section{(Un-)Gewöhnliches aus Ihrer Praxis}

Wie gefallen Ihnen unsere Geschichten aus der Praxis (s. 27)? Haben Sie in Ihrer Praxis auch Erlebnisse gehabt, die zum Schmunzeln oder Nachdenken anregen? Wollen Sie Ihre Geschichte mit unseren Lesern teilen? Dann schreiben Sie an: Brigitte.Moreano@springer.com. Jeder veröffentlichte Text wird honoriert. 